\title{
The Nobel Prize in Physiology or Medicine 2009 "for telomere biology" and its relevance to cancer and related diseases
}

\author{
Rosario Perona
}

$\mathrm{E}$ lizabeth H. Blackburn, Carol W. Greider and Jack W. Szostak have been jointly awarded the 100th Nobel Prize in Medicine and Physiology for the discovery of "how chromosomes are protected by telomeres and the enzyme telomerase".

This discovery of basic research done in a primitive organism has been a breakthrough for the understanding of how cells successfully maintain cell division and turn into tumoral or senescent cells, with an important impact in human health and disease. Telomeres were first described in 1930 by Hermann Müller (Nobel Prize 1945) and Barbara McClintock (Nobel Prize 1983) who observed in the fruit fly (Drosophila melanogaster) [1] and in maize (Zea mays) [2] that natural chromosome ends had special properties and structures that played an essential function in their protection. These structures were called telomeres (from the Greek "telos" for end and "melos" for part) by Müller. Later, it was known that telomeres are terminal DNA-protein complexes that form capping structures in order to stabilise chromosome ends and prevent them from being recognised as DNA double-strand breaks [3].

Later on, in 1961, Leonard Hayflick developed the concept of cell immortality by discovering that human cells undergo a limited number of cell divisions, demonstrating the finite replicative capacity of normal human fibroblasts [4]. The phenomenon was known as replicative senescence or the Hayflick limit. In the 1970s James Watson (Nobel Prize 1962) reported that the properties of DNA replication prevent the cell from fully copying their linear chromosomes up to the ends [5]. Replication takes place in the 5'-3' direction and requires RNA primers. This poses a

R. Perona ( $\square)$

Instituto de Investigaciones Biomédicas CSIC/UAM

C/ Arturo Duperier, 4

ES-28029 Madrid, Spain

e-mail: RPerona@iib.uam.es

R. Perona

CIBER de Enfermedades Raras (CIBERER)

Valencia, Spain problem for the initiation of replication at the very end of the chromosomes. Watson called this the "end-replication problem". Alexei Olovnikov found a link between this endreplication problem and cellular senescence (described by Hayflick). He postulated that the consecutive shortening of the DNA molecule in each round of replication might explain why normal cells can only divide a specific number of times and this can explain the Hayflick limit $[6,7]$.

In 1978 Elisabeth Blackburn, working in Joseph Gall's laboratory, found that telomeres from the ciliated protozoan Tetrahymena thermophila were formed by simple repeats of a 6-base pair fragments with the sequence TTGGGG, which protected the chromosomes from degradation [8]. Later on, Elizabeth Blackburn and Jack Szostak showed that the terminal repeated sequence of Tetrahymena could protect a linear plasmid from degradation in yeast [9]. Therefore, these sequences conferred telomere function. In 1885 Carol Greider and Elizabeth Blackburn discovered an enzyme that elongated telomeres and would compensate for the incomplete replication of telomere ends, allowing cells to divide without losing telomere repeats, and called this enzyme telomere terminal transferase [10]. They purified the enzyme and showed that it was a ribonucleoprotein complex, and both the RNA (hTR) and the protein (hTERT) were required for enzymatic activity [11], and gave this new type of DNA polymerase the name telomerase. Although this discovery was made in Tetrahymena, it was shown later that telomerase activity is the main mechanism by which telomeres are elongated in eukaryotes. J. Szostak, together with V. Lunbland, linked telomere shortening with the onset of cell senescence in yeast [12]. In parallel, researchers working on animal cells (C.B. Harley) [13] proposed that the Hayflick limit was due to an excessive telomere shortening leading to cell senescence because of the lack of telomerase in somatic cells. In contrast, germline cells have telomerase activity and are able to maintain telomere length, thus bypassing senescence. In was in 1994 when a PCR-based TRAP assay for telomerase activity was developed, thus allowing detection of telomerase in a vast majority of human cancer cells, pointing to telomerase as a potential target for anticancer therapy [14]. An animal 
model generated later on, a knockout mouse for the telomere RNA component (M. Blasco, 1977) [15], showed that telomerase was the main activity responsible for maintaining telomere length in mammals. In the absence of telomerase, mice suffered an accelerated rate of telomere shortening associated with ageing, age-associated pathologies and reduced longevity [16].

In 1990, a telomerase-alternative mechanism of maintaining telomeres was described in yeast. A few years later, in 1994, the mechanism was described in mammals. This mechanism was named 'alternative lengthening of telomeres' (ALT) [17]. After all these pivotal discoveries, intensive research was developed in many laboratories and outstanding results were obtained in different aspects of telomere biology: telomerase composition assembly and regulation, composition of telomeric chromatin, drug development and telomere-independent functions of telomerase.

Certainly all these findings have represented important advances in the understanding of cancer and, more recently, other human diseases. Short telomeres are a barrier for cell division and, in agreement with this, most cancer cells activate telomerase during tumorigenesis to maintain a minimal telomere length (even if telomeres are not further elongated). Telomerase reactivation is known to be one of the landmarks of oncogenesis, as defined by R. Weinberg [18]. Some tumour cells amplify both, telomerase genes, hTR and hTERT, in order to support tumour growth. Different strategies have been employed to inhibit telomerase in all kinds of tumour cells by targeting either hTR or hTERT or even the telomeres $[19,20]$. However, although much effort has been concentrated in this task, few compounds are exploited clinically, mainly for reasons of low effectiveness or in vivo toxicity. Because hTERT is immunogenic, it represents also a suitable target for cancer immunotherapy. Initial clinical trials of multiple vaccine formulations containing peptide directed against hTERT epitopes suggest that an immunoprevention therapy for cancer might be possible [21].

The implication of telomerase deficiency in different syndromes has represented an important advance in their knowledge and a potential therapeutic approach for such diseases. Deficiency in telomerase activity has been reported in haematopoietic syndromes such as dyskeratosis congenita, aplastic anaemia and, more recently, in different malignancies and myelodysplastic syndromes [22-24]. Mutations in either hTR or hTERT, or other components of the telomerase complex, such as dyskerin, Nola2 and Nola3, have been found in these groups of patients [23, 25]. In other diseases, such as idiopathic pulmonary, liver and heart fibrosis, whose origin is chronic inflammation in at least some patients, mutations in either hTR or hTERT were also found [26, 27].

Besides the genetic background, non-genetic environmental influences can impact on telomerase and telomere maintenance. This is the case for inflammatory processes such as ulcerative colitis, and for obesity, cardiovascular disease, hypertension and chronic exposure to cigarette smoke [28]. Determining the mechanisms by which telomerase activity is affected by external factors and by other metabolism and biochemical dysfunctions would help to better understand the role of telomerase in agerelated diseases. Recent reports from E. Blackburn's laboratory have shown that overproduction of glucocorticoids due to exposure to chronic psychological stress can compromise telomere maintenance and that exercise and diet control preserve telomere length and counteract chromosome instability that impact on tissue renewal and disease [29].

The scientific discoveries of the 2009 Nobel Prize in Physiology and Medicine are excellent examples of the benefits that basic scientific research can provide to applied and clinical research. These findings, although made in a simple organism such a Tetrahymena, have been shown to be pivotal in the understanding of diseases such as cancer, dyskeratosis congenita and other age-related diseases. Furthermore, they have provided opportunities for drug discovery of new therapies for both cancer and telomerase deficiency syndromes. In the future it would help to understand how environment and lifestyle factors can contribute to telomere-based human ageing diseases.

\section{References}

1. Müller HJ (1938) Bar duplication. Collecting Net (Woods Hole) 13:181-198

2. McClintock B (1939) The behavior in successive nuclear divisions of a chromosome broken at meiosis. Proc Natl Acad Sci U S A 25:405-416

3. McClintock B (1941) The stability of broken ends of chromosomes in Zea mays. Genetics 28:234282

4. Hayflick L, Moorhead PS (1961) The serial cultivation of human diploid cell strains Exp Cell Res 25:585-621

5. Watson JD (1972) Origin of concatemeric T7 DNA. Nat New Biol 239:197-201

6. Olovnikov AM (1971) Principle of marginotomy in template synthesis of polynucleotides. Dokl Akad Nauk SSSR 201:1496-1499

7. Olovnikov AM (1973) A theory of marginotomy. The incomplete copying of template margin in enzymatic synthesis of polynucleotides and biological significance of the phenomenon. J Theor Biol 41:181-190

8. Blackburn EH, Gall GJ (1978) A tandemly repeated sequence at the termini of the extrachromosomal ribosomal RNA genes in Tetrahymena. $\mathrm{J}$ Mol Biol 120:33-53

9. Szostak JW, Blackburn EH (1982) Cloning yeast telomeres on linear plasmid vectors. Cell 29:245255

10. Greider CW, Blackburn EH (1985) Identification of a specific telomere terminal transferase activity in Tetrahymena extracts. Cell 43:405-413

11. Greider CW, Blackburn EH (1987) The telomere terminal transferase of Tetrahymena is a ribonucleoprotein enzyme with two kinds of primer specificity. Cell 51:887-898

12. Lundblad V, Szostak JW (1989) A mutant with a defect in telomere elongation leads to senescence in yeast. Cell 57:633-643

13. Harley CB, Futcher AB, Greider CW (1990)
Telomeres shorten during ageing of human fibroblasts. Nature 345:458-460

14. Kim NW, Piatyszek MA, Prowse KR et al (1994) Specific association of human telomerase activity with immortal cells and cancer. Science 266:2011-2015

15. Blasco MA, Lee HW, Hande MP et al (1997) Telomere shortening and tumor formation by mouse cells lacking telomerase RNA. Cell 91:25-34

16. Blasco MA (2005) Telomeres and human disease: ageing, cancer and beyond. Nat Rev Genet 6:611-622

17. Bryan TM, Reddel RR (1997) Telomere dynamics and telomerase activity in in vitro immortalised human cells. Eur J Cancer 33:767-773

18. Hanahan D, Weinberg RA (2000) The hallmarks of cancer. Cell 100:57-70

19. Deville L, Hillion J, Lanotte M et al (2006) Diagnostics, prognostic and therapeutic exploitation of telomeres and telomerase in leukemias. Curr Pharm Biotechnol 7:171-183 
20. Pendino F, Tarkanyi I, Dudognon C et al (2006) Telomeres and telomerase: pharmacological targets for new anticancer strategies? Curr Cancer Drug Targets 6:147-180. Review

21. Vonderheide RH (2008) Prospects and challenges of building a cancer vaccine targeting telomerase. Biochimie 90:173-180

22. Mitchell JR, Wood E, Collins K (1999) A telomerase component is defective in the human disease dyskeratosis congenita. Nature 402:551-555

23. Mason PJ, Wilson DB, Bessler M (2005) Dys- keratosis congenita: a disease of dysfunctional telomere maintenance. Curr Mol Med 5:159-170

24. Hills M, Lansdorp PM (2009) Short telomeres resulting from heritable mutations in the telomerase reverse transcriptase gene predispose for a variety of malignancies Ann N Y Acad Sci 1176:178-190

25. Kirwan M, Dokal I (2009) Dyskeratosis congenita, stem cells and telomeres. Biochim Biophys Acta 1792:371-379

26. Armanios MY, Chen JJ, Cogan JD et al (2007) Telomerase mutations in families with idiopathic pulmonary fibrosis. N Engl J Med 356:1317-1326

27. Alder JK, Chen JJ, Lancaster L et al (2008) Short telomeres are a risk factor for idiopathic pulmonary fibrosis. Proc Natl Acad Sci U S A 105 : 13051-13056

28. Valdes AM, Andrew T, Gardner JP et al (2005) Obesity, cigarette smoking, and telomere length in women. Lancet 366:662-664

29. Epel ES, Blackburn EH, Lin J et al (2004) Accelerated telomere shortening in response to life stress. Proc Natl Acad Sci U S A 101:17312-17315 